\title{
WEAK DISJOINTNESS OF TRANSFORMATION GROUPS
}

\author{
REUVEN PELEG ${ }^{1}$
}

\begin{abstract}
Two transformation groups (t.g.) are called weakly disjoint if their product is ergodic. We characterize this relation for a certain class of t.g. and then prove that for $(X, T)$ and $(Y, T)$ in a certain family of t.g. $(X, T)$ and $(Y, T)$ are disjoint iff they have no nontrivial common factor. Finally, we generalize some disjointness relátions of [2] and [4].
\end{abstract}

\section{Weak disjointness.}

Definition. $(X, T)$ and $(Y, T)$ are weak!y disjoint if $(X \times Y, T)$ is ergodic. We denote this relation by $X-Y$. (We consider only t.g.'s on compact spaces.)

The reader is referred to [2] and [3] for concepts and notation.

Lemma 1. If $X$ is minimal and $Y$ is ergodic, then $X \perp Y$ implies $X-Y$.

Proof. Let $\Delta \subseteq X \times Y$ be a subflow with a nonempty interior. The minimality of $X$ and the ergodicity of $Y$ imply that the projections from $\Delta$ to $X$ and $Y$ are onto. Thus $\triangle$ must be equal to $X \times Y$ so that $X-Y$.

The next lemma is merely a reformulation of definitions.

LeMma 2. $X$ is weakly mixing iff $X \dot{-} Y$.

In order to characterize the relation $X-Y$ we use a method of [1] which was used later in [3], but first we need two lemmas.

LeMMA 3. If $(X, \mu),(Y, v)$ are probabiiity spaces and $(Y, v)$ is separable then the following conditions on a measurable subset $E \subset X \times Y$ are equivalent:

(1) $E$ is a rectangle (a.e. $\mu \times v)$ of the form $X \times B$.

(2) $v\left(E_{x}\right)=C$ a.e. $\mu$ and $v\left(E_{x} \cap E_{s}\right)=C^{\prime}$ a.e. $\mu \times \mu$ ( $E_{x}$ denotes the section at $x$ ).

Received by the editors April 6, 1971.

AMS 1969 subject classifications. Primary 5482.

Key words and phrases. Transformation groups, disjointness, eigenvalues.

1 This work was done under the supervision of Professor H. Furstenberg as a part of the author's expected Ph.D. thesis to be submitted to the Hebrew University of Jerusalem.

c American Mathematical Society 1972 
Proof. $\quad(1) \Rightarrow(2)$ is obvious.

Assume (2). Let $X_{1}=\left\{x: v\left(E_{x}\right)=C\right\}, F=\left\{x, s \mid v\left(E_{x} \cap E_{s}\right)=C^{\prime}\right\}$. Choose a dense sequence $\left\{A_{i}\right\}$ in the metric space of measurable sets of $Y$ and define, for an arbitrary $\varepsilon>0, \Omega_{i}=\left\{x: v\left(A_{i} \cap E_{x}\right)<\varepsilon\right\} \cap X_{1} . X_{1}=\bigcup_{i} \Omega_{i}$ so we may find a set $\Omega_{i 0}$ with positive measure. For any $(x, s) \in\left(\Omega_{i 0} \times \Omega_{i 0}\right) \cap F$, $v\left(A_{i 0} \triangle E_{x}\right)<\varepsilon, v\left(A_{i 0} \triangle E_{s}\right)<\varepsilon, v\left(E_{x}\right)=v\left(E_{s}\right)=C$, and $v\left(E_{x} \cap E_{s}\right)=C^{\prime}$. Thus

$$
2 \varepsilon>v\left(E_{x} \cap E_{s}\right)=v\left(E_{x}\right)+v\left(E_{s}\right)-2 v\left(E_{x} \cap E_{s}\right)=2\left(C-C^{\prime}\right) .
$$

Since $\varepsilon$ was arbitrary, we have $C=C^{\prime}$.

Now the set $\left(X_{1} \times X_{1}\right) \cap F$ has measure 1 and for any $(x, s)$ in it $v\left(E_{x} \triangle E_{s}\right)=0$. This implies (1).

REMARK. A similar proof, using the separability of $L_{2}(Y, v)$ yields a sufficient and necessary condition for a function in $L_{2}(X \times Y, \mu \times v)$ to be in $L_{2}(Y, v)$. We do not know whether the assumption of separability is essential.

LEMMA 4. If $\mu$ is a closed ergodic invariant measure supported by the metric t.g. $(X, T), v$ is an invariant measure supported by $(Y, T)$ and $E \subset X \times Y$ is closed invariant, then $v\left(E_{x}\right)$ is constant a.e.

Proof. $v\left(E_{x}\right)=\int k(x, y) d v(y)$ where $k$ is the characteristic function of $E$. $k(x, y)$ is upper semicontinuous so $v\left(E_{x}\right)$ has the same property. Thus, for every $\alpha, A_{x}=\left\{x: \nu\left(E_{x}\right) \geqq \chi\right\}$ is closed. $A_{\alpha}$ is a closed invariant set, so its measure is 0 or 1 . This implies that $\nu\left(E_{x}\right)$ is constant a.e.

We turn now to settle the abelian case. In the next theorem $X$ may be any separable metric Baire space.

THEOREM 5. If $T$ is abelian, $(X, T)$ and $(Y, T)$ are t.g. which support closed ergodic invariant measures $\mu, v$ respectively, then $X-Y$ iff the only common eigenvalue of $(X, T)$ and $(Y, T)$ is 1 .

(See [3] for the definitions of eigenvalue and eigenfunction.)

Proof. If $(X, T)$ and $(Y, T)$ have a common eigenvalue $\lambda \neq 1$ with the eigenfunctions $f$ and $g$ respectively, then $f \bar{g}$ is a nonconstant invariant function of $X \times Y$. This is impossible when $X \dot{-} Y$ so the condition is necessary. We proceed to show sufficiency. Let $E \subseteq X \times Y$ be a subflow with a nonempty interior. We want to show $E=X \times Y$. Let $k(x, y)$ be the characteristic function of $E$ and $K(x, s)=\int k(x, y) k(s, y) d v(y)$. By Lemmas 4 and 3 it is sufficient to prove that $K(x, s)$ is constant a.e. $\mu \times \mu$. Assume the converse and define an operator $R$ on $L_{2}(x, \mu)$ by

$$
R f(x)=\int K(x, s) f(s) d \mu(s) .
$$


$R$ is a Hilbert Schmidt operator and just as in [1] and [3, Theorem 2.5], we deduce the existence of a nonconstant function $f \in L_{2}(x, \mu) \cap B(X)$ which satisfies $R f=\lambda$ f for some $\lambda \neq 0$ and $t f=\chi(t) f$ where $\chi$ is a character of $T$.

Define $g(y)=\int k(x, y) f(x) d \mu(x)$. Obviously, $g \in L_{2}(Y, v) \cap B(Y), \operatorname{tg}=$ $\chi(t) g$ and $g$ is not identically 0 because $\lambda f(x)=\int k(x, y) g(y) d v(y)$.

Thus, we found a common eigenvalue $\chi(t) \not \equiv 1$ so $K(x, s)$ must be constant a.e. $\mu \times \mu$.

Corollary 6. If $T$ is abelian, $(X, T)$ and $(Y, T)$ minimai, then $X \dot{-} Y$ iff $\gamma(X) \perp \gamma(Y) .(\gamma(X)$ is the maximal equicontinuous factor of $X$.

We turn now to the nonabelian case. If $G$ is a group of unitary matrices of order $n \times n$ and $\alpha=\left(\alpha_{1}, \cdots, \alpha_{n}\right)$ is an $n$-tuple of complex numbers, then taking the orbit closure $\operatorname{cls}[\{\alpha A \mid A \in G\}]$ we get a minimal isometric transformation group.

Definition. If $(X, T)$ is a right t.g., $f_{1}, \cdots, f_{n} \in B(X)$ are independent, then $\left(f_{1}, \cdots, f_{n}\right)$ is called n-eigenfunction ( $n$-e.f.) if there exists an antihomomorphism $\chi$ from $T$ onto a group $G$ of unitary matrices which satisfies: $t\left(f_{1}, \cdots, f_{n}\right)=\left(f_{1}, \cdots, f_{n}\right) \chi(t)$ for a comeager subset of $X . \chi$ is called an n-eigenvalue (n-e.v.). Recall Theorem 2.2 of [2]:

LEMMA 7. $(X, T)$ is ergodic iff 1 is a simple l-e.v.

LEMMA 8. If $F$ is an n-eigenfunction and $U \subset X$ is comeager, then there exist $x_{1}, \cdots, x_{n} \in U$ such that $\left\{F\left(x_{i}\right)\right\}$ is a basis of $C^{n}$.

Proof. Let $L$ be the subspace spanned by $\{F(x) \mid x \in U\}$. If $L$ is not the entire space one can find a vector $\alpha \neq 0$ such that the inner product $(\alpha, F(x))=0(x \in U)$. This contradicts the independence of $\left(f_{1}, \cdots, f_{n}\right)=F$.

THEOREM 9. If $(X, T)$ and $(Y, T)$ are t.g. supporting closed ergodic invariant measures $\mu, v$ respectively, then $X \dot{-} Y$ iff their only common $n$ eigenvalue is the 1-e.v. 1.

Proof. The condition is necessary. Let $\chi(t)$ be a common $n$-e.v., $n>1$ (the case $n=1$ is simple). Let $F$ and $G$ be $n$-e.f. which correspond to $\chi(t)$. Let $\phi(x, y)=\langle F(x), G(y)\rangle$ then

$\phi(x t, y t)=\langle F(x t), G(y t)\rangle=\langle F(x) \chi(t), G(y) \chi(t)\rangle=\langle F(y), G(y)\rangle=\phi(x, y)$

(the author is indebted to the referee for this simple proof of the invariance). We proceed to show that this invariant function is not constant on a comeager set $A \subset X \times Y$. Assume the contrary. There exists a comeager set $V \subset Y$ such that for every $Y \in V$ the section $A y$ of $A$ is comeager in $X$. Let $\left\{G\left(y_{j}\right)\right\}, y_{1}, \cdots, y_{n} \in V$, form a basis of $C^{n}$ and choose $x_{1}, \cdots, x_{n} \in$ $\bigcap_{1}^{n} A y_{i}$ such that $\left\{F\left(x_{j}\right)\right\}$ is another basis of $C^{n}$. Let $0 \neq \gamma \in C^{n}, \sum \gamma_{j}=0$ and 
choose $\left\{\delta_{j}\right\}_{1}^{n}$ to satisfy $v=\sum_{1}^{n} \gamma_{j} F\left(x_{j}\right)=\sum_{1}^{n} \delta_{j} G\left(y_{j}\right)$ then $0<\langle(v, v)\rangle=0$ shows the contradiction.

The sufficiency is proved essentially in the same way as in Theorem 5 . Let $\left(f_{1}, \cdots, f_{n}\right)$ be an orthonormal basis for the space of eigenfunctions of $R$ which correspond to $\lambda$. There exists then a group of unitary matrices $\chi(t)$ such that $t\left(f_{1}, \cdots, f_{n}\right)=\left(f_{1}, \cdots, f_{n}\right) \chi(t)$. We define $\left(g_{1}, \cdots, g_{n}\right)$ as in Theorem 5 and find that $\chi$ is a common $n$-eigenvalue.

Lemma 10. If $(X, T)$ is minimal, then every n-e.v. $\chi(t)$ defines an equicontinuous factor of $(X, T)$.

Proof. If $F=\left(f_{1}, \cdots, f_{n}\right)$ is an $n$-e.f., then $F$ must be continuous (as in $[1$, p. 506]). Thus $x \rightarrow F(x)$ defines a homomorphism between $(X, T)$ and $(F(X), \chi(T))$.

Now we have the analogue of Corollary 6 .

THEOREM 11. If $(X, T)$ and $(Y, T)$ are minimal t.g. supporting invariant measures $\mu$ and $v$, then $X-Y$ iff $\gamma(X) \perp \gamma(Y)$.

Disjointness relations. If $X$ is a minimal flow we denote by $\operatorname{PD}(X)$ all the minimal metric flows built from $X$ by successive proximal and distal extensions and inverse limits. (In a proximal extension every two points in the same fiber are proximal; in a distal extension no two such points are proximal.)

The following theorem generalizes an unpublished result of Ellis.

THEOREM 12. Let $T$ be abelian, $X, Y$ minimal with $X \perp Y$. If $X^{\prime} \in \operatorname{PD}(X)$ and $Y^{\prime} \in \mathrm{PD}(Y)$, then $X^{\prime} \perp Y^{\prime}$ iff they have no nontrivial common factor.

Proof. It follows from [5] that proximal extensions preserve disjointness in the abelian case, so we have to take care only for the distal extensions. But if $X_{z} \perp Y_{\alpha^{\prime}}$, and $X_{x+1}$ is a distal extension of $X_{x}$ which has no common factor with $Y_{x^{\prime}}$, then by Corollary 6, $X_{\alpha+1} \perp Y_{\alpha^{\prime}} . X_{x+1} \times Y_{x^{\prime}}$ as a distal extension of $X_{x} \times Y_{x^{\prime}}$ which is minimal is semisimple too. So it must be minimal and $X_{\alpha+1} \perp Y_{x^{\prime}}$. The proof is completed by induction.

Lemma 13. Let $X$ and $Y$ be minimal t.g. If $X$ is distal and it is disjoint from the maximal distal factor of $Y$ then $X \perp Y$.

Proof. Let $D$ be the universal distal t.g., $z$ the distal part of $Y$ and $\phi: D \rightarrow X \times Z$ a homomorphism. Now we have the following diagram:

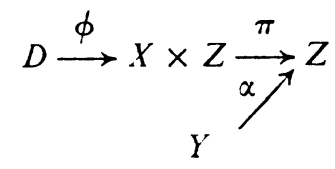


( $\pi$ is the projection.) By [7], $\{(d, y) / \pi \phi(d)=\alpha(y)\}$ is minimal. Therefore, $\{(x, z, y) / z=x(y)\}$ is minimal and so $X \times Y$ is minimal too.

TheOREM 14. Let $X$ and $Y$ be metric minimal t.g., $X$ distal. Then $X \perp Y$ iff $\gamma(X) \perp \gamma(Y)$.

Proof. Distal minimal t.g. always support an invariant measure ([1], [5]) so if $Z$ is the distal part of $Y$ then, by Theorem $11, X \perp Z$ iff $\gamma X \perp \gamma Z$ and the preceding lemma finishes the proof.

Next, we want to get some disjointness relations for a general $T$. If $A$ is any family of t.g. with phase group $T$, then $A_{I}$ will denote those t.g. of $A$ which support an ergodic measure. $\mathscr{M}, \mathscr{L}, \mathscr{E}$, and $\mathscr{W}$ denote respectively, the minimal, distal, ergodic, and weakiy mixing t.g.

Lemma 15. If $X, Y \in \mathscr{E}_{I}$ and $X-Y$, then $X \times Y \in \mathscr{E}_{I}$.

Proof. The proof of Theoren 9 shows that $\mu \times v$ is a closed ergodic invariant measure supported by $X \times Y$. The lemma follows now by Proposition 2.6 of [3].

The next theorem generalizes the corresponding results of [2] and [4]:

THEOREM 16. (1) $\left(\mathscr{M}^{-}\right)_{I}=\mathscr{H}^{`}{ }_{I}$.

(2) $\mathscr{W}_{I} \times \mathscr{H}_{I}^{-}=\mathscr{W}^{\circ}$.

(3) $X \in \mathscr{H}^{\circ}{ }_{1} \Rightarrow X^{n} \in \mathscr{W}^{\circ}$.

(4) $\mathscr{W}_{I}-\mathscr{E}_{I} \cup \mathscr{M}$

(5) $\mathscr{W}_{I} \perp \mathscr{I} \cap \mathscr{M}$

(6) $\left(\mathscr{L}^{-}\right)_{I}=\mathscr{W}^{\circ} \cap \mathscr{M}_{1}$.

Proof. (1) and (2) follow by Lemma 15 and Theorem 9. (3) is a special case of (2).

(3) implies that if $X \subseteq \mathscr{H}_{I}$ then $N(A, B)$ contains a left translation of every finite subset of $T$. This implies $\mathscr{W}_{I}-\mathscr{M} . \mathscr{W}_{I}-\mathscr{E}_{I}$ follows from Theorem 9 and thus we established (4).

For (5) use [4] and Furstenberg's structure theorem as in [2, Theorem 3].

(6) is a consequence of (4) and (5) (see Theorem 4.4 in [4]).

REMARK. The subscript $I$ is essential. If we take $X$ to be the unit circle and $T$ is generated by the two homeomorphisms

$$
\exp 2 \Pi i x \rightarrow \exp 2 \Pi i(x+\Pi) \text { and } \exp 2 \Pi i x \rightarrow \exp 2 \Pi i x^{2},
$$

then $(X, T) \in \mathscr{W}^{\prime}$ but $X \times X \times X \notin \mathscr{E}$.

Our last theorem is independent from the rest of the paper and gives some more information about $(\mathscr{W} \cap \mathscr{M})^{\perp}$. Call $(X, T)$ weakly distal if there exists an $x_{0} \in X$ with card $x_{0} P(X) \leqq \aleph_{0} .(P(X)$ is the proximal relation of $X$.) 
THEOREM 17. If $(X, T)$ is a weakly distal minimal metric abelian t.g. then $X \perp \mathscr{H} \cap \mathscr{M}$.

Proof. Let $Y \in \mathscr{H} \cap \mathscr{H}$ and let $\Delta$ be a minimal subflow of $X \times Y$. We have to show $\Delta=X \times Y$. Choose $\left(x_{0}, y_{0}\right) \in \Delta$ with card $x_{0} P(X) \leqq \aleph_{0}$ and denote by $\pi$ the projection from $\Delta$ onto $Y$. By [6], $y_{0} P(Y)=A$ is a residual set and, since $\pi\left[\left(x_{0}, y_{0}\right) P(\Delta)\right]=A$, we can find for every $y \in A$ an $x_{i} \in x_{0} P(X)$ so that $\left(x_{0}, y_{0}\right)$ and $\left(x_{i}, y\right)$ are proximal in $\Delta$. Define

$$
B_{i}=\operatorname{cls}\left\{y:\left(x_{0}, y_{0}\right\rangle,\left\langle x_{i}, y_{\rangle}\right) \in P(\Delta)\right\} .
$$

Obviously, $\left\{x_{i}\right\} \times B_{i} \subset \Delta$ and $\bigcup B_{i} \supset A$ so that we may find a $B_{j}$ with a nonempty interior. Let $U \times V$ be an open rectangle in $X \times Y . N\left(x_{j}, U\right)$ is discretely syndetic and $N\left(B_{j}, V\right)$ is discretely replete so that $N\left(x_{j}, U\right) \cap$ $N\left(B_{j}, V\right) \neq \varnothing$. Thus, the orbit of $\left\{x_{j}\right\} \times B_{j}$ is dense and $\Delta=X \times Y$.

Appendix. Using the remark after Lemma 3 and the technique of Theorem 5 , one can show that if $(X, T, \mu)$ and $(Y, T, v)$ are ergodic processes, then every eigenvalue of $(X \times Y, T, \mu \times v)$ is the product of an e.v. of $X$ with an e.v. of $Y$. Furthermore, if $\mu \times v$ is ergodic, then every e.f. of $X \times Y$ is the product of an e.f. of $X$ with an e.f. of $Y$.

\section{REFERENCES}

1. H. Furstenberg, The structure of distal flow:s, Amer. J. Math. 85 (1963), 477-515. MR 28 \#602.

2. - Disjointness in ergodic theory, minimal sets, and a problem in Diophantine approximation, Math. Systems Theory 1 (1967), 1-49. MR 35 \#4369.

3. H. B. Keynes and J. B. Robertson, Eigenvalue theorems in topological transformation groups, Trans. Amer. Math. Soc. 139 (1969), 359-369. MR 38 \#6029.

4. H. B. Keynes, The structure of weakly mixing transformation groups (preprint).

5. R. Ellis, Lectures on topological dynamics, Benjamin, New York, 1969.

6. R. Ellis and H. Keynes, A Characterization of the equicontinuous structure relation (preprint).

7. L. Shapiro, Distal and proximal extensions of minimal flows, Math. Systems Theory 5 (1971), 76-88.

Department of Mathematics, Hebrew University, Jerusalem, Israel 